\title{
La institucionalización de la Ciencia en Mendoza y la región de Cuyo (1948-1957)
}

\section{El caso del Departamento de Investigaciones Científicas (DIC) de la Universidad Nacional de Cuyo ${ }^{1}$}

\author{
The Institutionalization of Science in Mendoza and the Cuyo region \\ (1948-1957). The case of the Department of Scientific Investigations \\ (DIC) of the Universidad Nacional de Cuyo
}

PABLO ANTONIO PACHECO

Universidad Nacional de Cuyo

RESUMEN Las instituciones evidencian procesos de institucionalización de las ciencias, como cristalización de prácticas, actividades y discursos científicos. Ponen en juego relaciones de poder, determinan métodos de trabajo, modos de transferencia del conocimiento y fines de la actividad científica, por constituir expresiones de estructuras económicas, sociales e ideológicas.

El presente trabajo se propone, a partir de fuentes documentales, testimonios y entrevistas, describir las actividades del Departamento de Investigaciones Científicas (DIC) de la Universidad Nacional de Cuyo, y analizar sus consecuencias para el proceso regional de institucionalización de la ciencia entre 1948 y 1957.

Palabras clave Departamento de Investigaciones Científicas - Centro de Estudios Físico Matemáticos institucionalización de la Ciencia - peronismo - ciencia pura

\begin{abstract}
The institutions show the process of the institutionalization of science as a crystallization of practices, activities and scientific discourses. They put into play the power relations involved, determine working methods, knowledge transfer modes and purposes of scientific activity, to constitute expressions of economic, social and ideological structures.

This paper proposes, from documentary sources, testimonies and interviews, to describe the activities of the Department of Scientific Investigation (DIC) of the National University of Cuyo, and to analyze their implications for the regional process of the institutionalization of science between 1948 and 1957.
\end{abstract}

Key words Department of Scientific Research - Centre for Mathematical Physics Studies - institutionalization of science - peronism - pure science 


\section{Introducción}

En el marco de los Estudios Sociales de la Ciencia y la Tecnología, el análisis de las instituciones constituye un abordaje fundamental para la comprensión de los procesos de institucionalización de las ciencias, como cristalización de prácticas, actividades y discursos científicos. Ellas ponen en juego relaciones de poder, determinan métodos de trabajo, modos de transferencia del conocimiento y fines de la actividad científica, al constituirse en expresiones de estructuras económicas, sociales e ideológicas. ${ }^{2}$

Los esfuerzos por desarrollar y consolidar una cultura científica en Mendoza y la región de Cuyo, se incrementaron desde finales de 1940, con la creación de diversas instituciones de carácter científico. Los intentos de planificación de la política científica en el ámbito universitario local, se concentraron en la estrategia de organizar un Departamento de Investigaciones Científicas (DIC), en función de la combinación de factores propios del contexto internacional de posguerra y de los intereses de la industrialización nacional.

La creación de dicho Departamento en el ámbito de la Universidad Nacional de Cuyo, constituye un elemento fundamental para comprender tanto la política de investigación científica implementada por los funcionarios universitarios, como el proceso local de consolidación de diversos campos disciplinares durante el período que va desde 1948 hasta el momento en que el instituto científico fue disuelto en 1957.

Diego Hurtado de Mendoza y Analía Busala, señalan dos aspectos críticos en la producción académica sobre la ciencia y la técnica durante el primer y segundo gobierno peronista. Para estos autores:

Dos razones se complementan, a nuestro juicio -una de orden general vinculada a un sesgo de la tradición historiográfica argentina, la otra vinculada al proceso histórico específico-, para explicar esta ausencia: (i) la poca atención que la historiografía argentina ha prestado, en general, al desarrollo científico y tecnológico; (ii) la aceptación tácita en las últimas dos décadas de una versión fundada en testimonios de protagonistas y en unas pocas obras de escaso rigor histórico, que sostiene que las bases del actual sistema científico y tecnológico argentino fueron concebidas y comenzaron a edificarse a partir de 1956." ${ }^{{ }^{3}}$

Por otra parte, las referencias que existen son aisladas y no precisan detalles ni profundizan la comprensión de conjunto de las actividades del organismo de investigación. Sin desconocer el valor que tienen estos trabajos ${ }^{4}$ tanto por los criterios de interpretación del período que ofrecen como por ser los pocos que lo abordan, ninguno de los estudios consultados trata de manera sistemática el análisis del Departamento de Investigaciones Científicas de la Universidad Nacional de Cuyo en su totalidad, que es el objeto de este trabajo. El tratamiento es general o centrado en las actividades del Instituto de Matemática, que tuvo reconocimiento internacional. ${ }^{5}$ Por ejemplo, al ilustrar una modalidad de institucionalización de la investigación científica negadora de la institucionalidad y asentada en el factor humano, Jorge Myers afirma: "Otro caso perteneciente a la disciplina matemática, que se conformara según este modelo institucional, es el Departamento de Investigaciones Científicas de la Universidad Nacional de Cuyo, fundado en 1952. La sección Matemática de esta institución convertiríase durante tres años en uno de los centros más importantes de investigación matemática". 6

Los autores refieren aspectos aislados del Departamento, en el conjunto de iniciativas del período, probablemente debido a la falta de acceso a la documentación que enmarca la creación de este organismo. En tal sentido, resulta necesaria una reconstrucción de las actividades del Departamento y de todas sus secciones, para una comprensión más amplia de las implicancias y consecuencias que tuvo la iniciativa de la universidad cuyana durante aquellos años.

El presente trabajo aborda la descripción del contexto intelectual, científico e institucional previo a la creación del DIC, para concentrarse luego en la organización de ese departamento, destacando las actividades científicas más relevantes desarrolladas en el mismo. De este modo, a partir de fuentes documentales, periodísticas, entrevistas y relatos personales, el texto pretende ser un aporte necesario a la comprensión histórica de las modalidades asumidas por las actividades y la política científica en la región durante ese período. 
Se avanzan como consideraciones finales, en primer lugar la amplitud y complejidad del objeto de estudio, atravesado por factores económicos, sociales, políticos y culturales que operaron durante ese tiempo. En segundo lugar, las implicancias de dicho objeto para la institucionalización científica, evidencian su inclusión en una modalidad "negadora de la institucionalidad", 7 que tuvo sus consecuencias en campos como los de la física nuclear, las matemáticas y la biología. En tercer lugar, el objeto se sitúa como antecedente para la conformación de un complejo científico y tecnológico nacional, en el marco de la política científica del gobierno peronista (1945-1955).

\section{Ciencia, universidad e industrialización}

La respuesta industrializadora de la oligarquía terrateniente argentina a la crisis de 1929-1930, ${ }^{8}$ tuvo consecuencias para las actividades científicas. Implicó un reajuste de las relaciones entre la universidad, el incipiente complejo científico tecnológico, y el sistema productivo, ${ }^{9}$ bajo la llamada "movilización industrial". ${ }^{10}$ Aunque en algunas áreas de la ingeniería como la aeronáutica o la navegación existieron desarrollos autónomos importantes, desde 1940 se produjo un movimiento de transferencia de modelos y matrices básicas de organización de ciencia y tecnología, desde Europa Occidental a los países latinoamericanos. ${ }^{11}$

La Segunda Guerra Mundial promovió en los países centrales, una política de cooptación de científicos y técnicos provenientes de Alemania e Italia y otros países europeos afines al nazismo y al fascismo. En Latinoamérica, tanto Brasil como Argentina, siguieron una "política de transferencia", ofreciendo a profesores e investigadores alemanes e italianos, posibilidades para obtener cargos en distintas instituciones y universidades de ambos países. ${ }^{12}$ En nuestro país, esa política fue contracara de los conflictos entre gran parte de la comunidad científica local y los sectores alineados con el plan de gobierno. ${ }^{13}$

Las iniciativas oficiales para articular la docencia y la investigación en el ámbito universitario local, se enmarcaron en ese complejo contexto, insoslayable para valorar la significación que adquiere todo núcleo de actividad científica en el horizonte regional.

En Mendoza, los funcionarios universitarios inician un proceso de cambios en diversos niveles del sector, orientados al logro de los objetivos propuestos en la agenda nacional del primer Plan Quinquenal. La generación de "enclaves" intelectuales destinados a la investigación básica o "ciencia pura" como se le llamaba, aparecía como una exigencia de la alternativa de desarrollo económico planteada. Disciplinas que no mostraban vinculaciones directas con la industrialización pero cuyas investigaciones podían significar nuevas contribuciones al conocimiento en función de aplicaciones o desarrollos técnico-industriales, recibieron importante impulso. En tal sentido, la "ciencia pura" se consideraba una base fundamental para producir ciencia aplicada, desarrollar técnica y aportar a la industrialización. ${ }^{14}$ En el ambiente universitario mendocino la ciencia pura era entendida como actividad desinteresada, sin fines materiales, dedicada exclusivamente a generar nuevos aportes y contribuir al avance del conocimiento científico. Dicha actividad se consideraba relevante para ofrecer una base intelectual desde la cual desarrollar aplicaciones o técnicas industriales, idea que se sintetizaba en la expresión "ciencia técnica". Sería fundamental, aunque no es el objetivo de este artículo, determinar si esta manera de entender la actividad científica promovió u obstaculizó la posibilidad efectiva de desarrollos técnicos e industriales de relevancia.

Los discursos de funcionarios universitarios de la época evidencian ese nexo entre investigación básica, técnica e industria. En una intervención pública clave para entender la creación del DIc, el rector de esos años, Fernando Ireneo Cruz, sostenía: "Si nosotros no tenemos una ciencia pura representada por hombres y consagraciones, hombres al servicio de ese desinterés, seremos siempre subsidiarios, seremos siempre dependientes tanto en esa ciencia técnica y aplicada como en la industria y en la vida cultural". ${ }^{15}$

El proyecto de creación del Departamento de Investigaciones Científicas de la Universidad Nacional de Cuyo, se incorporó a los intentos por ensayar respuestas a la orientación del desarrollo económico. En tal sentido, con- 
tinuó la tendencia de transformaciones de las actividades universitarias, iniciada durante la fase de sustitución de importaciones para acomodar la investigación básica a las exigencias del desarrollo. ${ }^{16}$ Asimismo, el DIc se vinculó a los intereses de la defensa nacional en el marco de un proyecto geopolítico, que alentaba la colaboración entre la universidad y el ejército. Esto planteaba el desafío de superar la dependencia económica y cultural del país, desarrollando la investigación pura para ponerla al servicio de la ciencia aplicada, del progreso técnico y de la fuerza industrial y militar nacional. ${ }^{17}$

A fines de 1940, los cuadros intelectuales de la Universidad Nacional de Cuyo, asumieron el compromiso de generar ámbitos de investigación y divulgación científica, agrupándose en torno a distintas organizaciones con claro sentido de institucionalización de la ciencia. La universidad cuyana, junto con la Universidad Nacional de Tucumán, asumieron un rol central en el interior, para orientar la enseñanza y la política científica de acuerdo a los lineamientos planteados en los Planes Quinquenales de los dos primeros gobiernos de Perón. ${ }^{18}$

\section{Estrategias de institucionalización de la ciencia (1948-1950) $)^{19}$}

En la universidad cuyana, las Facultades de Ciencias de la Educación con sede en San Luis, Ingeniería y Ciencias Exactas, Físicas y Naturales con sede en San Juan, y Ciencias Económicas con sede en Mendoza, se crearon a principios de 1947. Las mismas, fueron desagregadas de la Facultad de Ciencias que las agrupaba y se les asignó presupuesto propio, interviniendo fuertemente en la conformación de un clima institucional para promover el desarrollo científico. ${ }^{20}$

A partir de 1948 se distinguen en Mendoza tres comunidades científicas claramente diferenciadas por sus actividades, desplegadas en torno a la universidad local. Una comunidad se formó alrededor del Departamento de Combustibles, que dependía de la mencionada Facultad de Ingeniería en San Juan, pero cuya sede se encontraba en Mendoza. Desde 1948, profesores de ese departamento organizaban periódicamente "sesiones de comunicaciones científicas", donde presentaban y discutían sus trabajos de investigación. En ese grupo se encontraban investigadores vinculados a la exploración y explotación de petróleo y minerales (Siegfried Lexow, Ernesto Maneschi, Armando Vergara Bai, Heriberto Windhausen, Ignacio González Arroyo, entre otros), así como a la física nuclear y la radiación cósmica (Otto Olindo María Gamba, ${ }^{21}$ Giovanni Pinardi). Además, este grupo organizó en 1948 un Museo Tecnológico y Laboratorios de Investigación para la formación y especialización de los estudiantes en la carrera de Ingeniero en Combustibles. El Museo Tecnológico y los Laboratorios constituyeron una estrategia para articular la investigación en ese departamento. La idea fue transferida de la iniciativa desarrollada por Gustavo Fester en la Universidad Nacional del Litoral (UNL) en la década de 1920, con quienes se habían formado un grupo de Químicos Industriales radicados posteriormente en Mendoza. ${ }^{22}$

Otra comunidad importante se organizó alrededor del Centro de Estudios Físico Matemáticos, creado el 27 de junio de 1948. Estuvo integrado por docentes de las tres facultades mencionadas y agrupaba a parte de los miembros del Departamento de Combustibles. La dirección del Centro consistía en una Comisión Coordinadora compuesta por tres miembros, uno por cada provincia cuyana sede (San Juan, San Luis y Mendoza), planteando desde el inicio una clara perspectiva regional. ${ }^{23}$ La institución funcionó hasta mediados de 1950, realizando seis reuniones de manera alternada en las tres provincias de Cuyo. Las exposiciones en los encuentros trataban sobre temas de astronomía, rayos cósmicos, física relativista, física nuclear, problemas de hidráulica, óptica, geodesia, sismología, álgebra, topología, lógica, contabilidad y hasta psicología del descubrimiento. Algunos de sus integrantes, participaron en la sección de Epistemología y Filosofía de la Naturaleza del Primer Congreso Nacional de Filosofía de 1949.24

El Centro de Estudios Físico Matemáticos y el Departamento de Combustibles organizaron en conjunto un curso de matemáticas. 
Una tercera comunidad orientada a impulsar la ciencia y su divulgación, se concentró en torno a una entidad denominada Asociación Científica de Mendoza, fundada el 3 de mayo de 1948 y cuyas actividades continuaron hasta 1951. A diferencia de las anteriores, esta institución no dependía de la universidad cuyana aunque varios integrantes eran docentes allí. Su finalidad era impulsar la investigación en Astronomía, Meteorología, Geofísica y Electrónica. Asimismo, la organización señaló en su primer comunicado de prensa, la conveniencia de vincularse con instituciones particulares o del Estado, para una mayor probabilidad de éxito de sus objetivos. ${ }^{25}$ Se conformó una mesa directiva con la estructura de una asociación civil, y un esquema organizativo que articulaba las actividades técnicas, de investigación, administrativas y de archivo. Esta asociación tuvo como base la incorporación efectiva, tanto de profesionales (profesores universitarios, médicos, ingenieros, contadores, militares) como de radioaficionados y aficionados a las disciplinas que promovía (comerciantes, productores agrícolas, empleados públicos), llegando a sumar un total de sesenta y dos miembros. ${ }^{26}$ Las actividades consistieron en la realización de conferencias de divulgación de las investigaciones y trabajos de los integrantes.

En el contexto tuvo también relevancia el Departamento de Ciencias Puras, posteriormente denominado Instituto de Ciencias Puras, ${ }^{27}$ organismo de investigación científica creado por la universidad a comienzos de 1948 y que dependía directamente del Rectorado. Para ocupar su dirección, las autoridades contrataron al doctor en Química italiano Giovanni Pinardi, como profesor interino de Termodinámica en el Departamento de Combustibles, y Director del Instituto de Ciencias Puras. ${ }^{28}$ Pinardi se doctoró en Torino y realizó estudios sobre radiación cósmica en Berna (Suiza), realizando exposiciones de placas nucleares en la Estación de Altura de la Testa Grigia (3.480 mts.), en el valle de Aosta (Italia), luego convertida en el Centro Nacional para el Estudio de la Física Nuclear.

A comienzos de 1948 Pinardi propuso a las autoridades el proyecto de emplazar una Estación de Altura en la zona de Laguna Diamante que funcionara como centro de física nuclear para el estudio de los rayos cósmicos. ${ }^{29}$ La obra se terminó de construir a comienzos de 1950 y mediante la colaboración de la Universidad y el Ejército se trasladó el instrumental científico y los víveres para quienes permanecerían en el refugio.

Los actores e instituciones mencionados, pusieron en práctica una serie de estrategias que buscaban desarrollar un ambiente científico:

- La selección de temas y problemas de investigación se asentó sobre la distinción fundamental y la conexión entre ciencia pura, ciencia aplicada y técnica.

- Se priorizaron temas vinculados a las posibilidades de explotación de recursos naturales y energéticos.

- Se desarrolló la investigación y la divulgación científicas como tareas que ampliaban y continuaban la actividad de la cátedra.

- Se conformaron ámbitos específicos para el intercambio de conocimientos (sesiones de comunicaciones científicas, seminarios, jornadas, cursos y conferencias).

- Se incorporaron tanto profesionales como aficionados a las actividades científicas, para salvar la brecha generada por la especialización del conocimiento, en un intento por instalar una particular visión humanista e integral de la ciencia.

- La pertenencia de varios actores a dos o más organizaciones constituyó una base para la vinculación entre instituciones.

- Se promovió un enfoque regional de las actividades científicas, para articular las tareas de investigación de los diferentes grupos de cada provincia cuyana. 
Estas estrategias de institucionalización de la ciencia, confluyeron hacia la organización y puesta en funcionamiento del Departamento de Investigaciones Científicas. De esta manera, el departamento se conformó a partir de la intensa actividad sostenida por los grupos intelectuales que animaron esas instituciones, y fue el resultado de la diversidad de prácticas científicas desarrolladas entre 1948 y 1950.

\section{Creación y organización del DIC}

Los miembros del Centro de Estudios Físico Matemáticos constituyeron la base para la creación del Departamento de Investigaciones Científicas de la Universidad Nacional de Cuyo en diciembre de 1949. La propuesta de esta iniciativa la realizó el rector Ireneo Cruz en la quinta reunión del Centro, el día 15 de diciembre por medio de un discurso ofrecido durante el desarrollo del encuentro. ${ }^{30}$

El proyecto tomó forma mediante la ordenanza $n^{0} 147$ del Consejo Universitario del 23 de diciembre de 1949, con sucesivas reorganizaciones entre febrero de 1950 y mayo de 1951, hasta su posterior desarticulación en octubre de 1957. ${ }^{31}$ La conformación de institutos de este tipo estaba legitimada por la Ley Universitaria 13.031.32

La prensa escrita local se hizo eco de la iniciativa y varios diarios de la época dieron a conocer partes de la disposición de creación y explicaron la organización y alcances que tenía el organismo. ${ }^{33}$ Asimismo, el 25 de diciembre de 1949 el rector Cruz ofrece una entrevista clave para entender el contexto, en donde expresa:

Las jornadas científicas realizadas en San Luis, que corresponden a otras realizadas en San Juan y Mendoza, provocan una intensa satisfacción en el actual gobierno universitario, porque constituyen, sin incentivo exterior alguno, la concentración de hombres de ciencia de la propia universidad, en las distintas pero comunes especialidades de las distintas facultades, que se ponen en contacto y en confrontación en trabajo de investigación científica, ofreciéndose a la crítica y ofreciéndose a un propio control de su estado de información y de su estado de capacidad de investigación. Cuando un conjunto de profesores apela a esta toma de conciencia científica y no teme el enfrentamiento con sus colegas en lides científicas de alto nivel, como ha ocurrido en todas las jornadas celebradas en la Universidad, hay mucho que esperar de las posibilidades de investigación pura de los hombres de esa Universidad. En este sentido, aproveché la circunstancia para proponer a los hombres de ciencia reunidos en estas jornadas un proyecto que consideramos fundamental para la vida universitaria del país; es decir, presenté a la consideración de los señores profesores la creación de un Departamento de Investigaciones Científicas que concentrará, en lo que atañe a la ciencia de la naturaleza, a todos los profesores con vocación científica definida y a todos los egresados y jóvenes profesores del país que deseen hacer cursos de investigación científica pura. ${ }^{34}$

Desde el principio el Dic fue concebido como un instituto de investigación con fuerte dependencia orgánica con el gobierno universitario. Así lo señala Cruz en su discurso: "Nuestro proyecto [... . queremos realizarlo nosotros aquí en forma orgánica, cohesionada y libre de todo vaivén político de las Facultades. Por eso propugnamos en este proyecto, que el Departamento dependa directamente del Rectorado". ${ }^{35}$ La sede se fijó en Mendoza por cuestiones administrativas, ${ }^{36}$ y su estructura estuvo integrada por el observatorio astronómico "Félix Aguilar" en San Juan y el Observatorio de Rayos Cósmicos en Mendoza, contando con las secciones de matemática, astronomía, física y biología. Se planteó la colaboración de los institutos, seminarios, laboratorios, bibliotecas y núcleos de investigación existentes en las facultades 0 que se creasen en el futuro. ${ }^{37}$

Se estableció un doble objetivo del organismo: por un lado, realizar investigaciones científicas y, por el otro, asumir la tarea de formación de investigadores. La finalidad con la que inicialmente fue proyectado a fines de la década de 1940, se mantuvo hasta su disolución, cuando fue cuestionada para intentar adaptarla a los intereses del golpe militar de $1955 .{ }^{38}$ 
El gobierno del Departamento estaba a cargo de un Consejo Técnico (CT) de diez miembros elegidos por el rector durante dos años, que debía reunirse una vez por mes en sesión ordinaria. El cт tenía como funciones redactar y modificar el reglamento de funcionamiento interno, disponer la creación de institutos, elaborar el presupuesto y administrar los fondos, realizar actividades de promoción de la actividad científica, organizar la enseñanza y los planes de estudio, proponer nuevos investigadores y aconsejar al rectorado sobre el otorgamiento de becas. Para formar parte del mismo se requería ser profesor o investigador de la universidad con "notoria dedicación científica", personal del Departamento 0 de otro instituto de la misma. ${ }^{39}$

La comisión encargada de dar los lineamientos del DIC estuvo conformada por Manuel Balanzat y Gabino F. Puelles en representación de la Facultad de Ciencias de la Educación en San Luis, Enrique Loedel Palumbo y Juan José Nissen por la Facultad de Ingeniería y Ciencias Exactas, Físicas y Naturales de San Juan, y Fausto Toranzos en representación de la Facultad de Ciencias Económicas de Mendoza. ${ }^{40}$

El cuerpo de docentes e investigadores del Dıc se constituyó con profesores que venían trabajando en la misma universidad o aprovechando la visita de investigadores nacionales y extranjeros, o bien mediante la contratación directa de recursos humanos, con una mayoría de científicos alemanes. ${ }^{41}$

La investigación se organizó en 1949 mediante la colaboración del personal docente de la universidad y la incorporación permanente 0 temporaria de personas destacadas en el campo de la ciencia, ${ }^{42}$ sin definirse inicialmente un escalafón hasta 1951, donde se establecieron cinco clases de investigadores: titulares, divididos en tres categorías (principal, asociado, y asistente), nombrados en forma definitiva y con dedicación exclusiva para investigación pura y formación de investigadores; auxiliares, opción para alumnos del último curso y egresados; incorporados, para el personal docente de la universidad; extraordinarios, para argentinos o extranjeros contratados; y correspondientes, dirigido a miembros investigadores de otras universidades o institutos nacionales o extranjeros que colaboraran con tareas específicas del Departamento. La selección de los investigadores debía hacerse mediante examen del currículo y dictamen fundamentado por escrito por una comisión designada por el ст. ${ }^{43}$ Cabe destacar que todos los investigadores del Departamento tenían cargos contratados con dedicación exclusiva, lo que tenía como finalidad promover e intentar consolidar la carrera de investigador científico.

El DIc otorgaría títulos de licenciado y doctor en ciencias en especialidades como matemática, astronomía, física, meteorología, aerofísica y química. La licenciatura se obtendría mediante la aprobación de los cursos, trabajos prácticos y un examen sin tesis, mientras que para el doctorado se establecía tener la licenciatura (o en caso contrario haber publicado en revistas trabajos de investigación que probaran la aptitud del aspirante) y la realización, impresión y distribución de una tesis original entre los miembros del tribunal evaluador. Los títulos serían conferidos por cada unidad académica respectiva a la especialidad. ${ }^{44}$

A principios de 1950 comenzaron a funcionar los primeros años de la licenciatura en matemática y licenciatura en física, mediante la incorporación de los cursos que dictaban varios profesores pertenecientes al Centro de Estudios Físico Matemáticos. El cт elaboró los primeros planes de estudio para las carreras de Meteorología y Aerofísica, en febrero de 1951. Los mismos tenían una duración de cinco años, constituidos por un trienio básico común a ambas y los dos últimos años con materias específicas obligatorias y optativas, al tiempo que los planes definitivos de matemática y física se encontraban en preparación. ${ }^{45}$ La estructuración interna de los ciclos comprendía tres secciones supervisadas por los profesores a cargo: la sección A introductoria a los estudios superiores donde "se hará una rigurosa selección", la sección B, de carácter "decisivo", si el alumno la aprobaba podía optar al grado de licenciado, de lo contrario no podía continuar, y finalmente la sección C posibilitaba optar al doctorado. ${ }^{46}$

Se estableció el desarrollo de los cursos según la materia y preferencias del profesor, con la sugerencia de evitar las clases magistrales e implementar seminarios o trabajos personales del alumno con lecturas y ejercicios de laboratorio, estableciendo cada instituto el puntaje, las correlatividades y obligatoriedad de los mismos. ${ }^{47}$

Como requisito de ingreso se dispuso ser ingeniero, profesor egresado o alumno con la totalidad de materias aprobadas en una facultad de la universidad, o egresado o alumno de otra universidad o instituto del profesorado 
mediante examen. En ambos casos, junto con la presentación de dos certificados de ex profesores que acreditaran la vocación científica. Además de un régimen estricto de selección, la situación de cada aspirante quedaba sujeta a evaluación por un comité. Se fijaba un cupo de veinte lugares con posibilidad de un examen si el número de aspirantes aumentaba, procurándose que la mayoría de alumnos fuesen becarios con dedicación exclusiva. ${ }^{48}$ Esto convertía al DIc en el instituto más "antidemocrático" de la universidad, característica reconocida por el rector en su discurso fundacional. ${ }^{49}$

Desde el punto de vista de la infraestructura y organización, entre diciembre de 1949 y julio de 1950, se iniciaron los trabajos para la construcción del observatorio de rayos cósmicos y la Estación de Altura en Mendoza, concretados en el lapso de seis meses..$^{50}$ También se creó un observatorio meteorológico en el Cerro Pelado (Mendoza) para los trabajos de aerofísica. ${ }^{51}$ El Observatorio "Félix Aguilar" fue inaugurado en septiembre de 1953, coincidiendo con jornadas científicas de la Asociación Física Argentina. ${ }^{52}$

Las inscripciones comenzaron en 1950, convocándose entre marzo y abril de cada año. Las actividades se desarrollaban en las diversas facultades de la universidad. ${ }^{53}$

\section{Institutos, actividades científicas y formación de investigadores en el DIC}

Las secciones y actividades desarrolladas en el Departamento de Investigaciones Científicas incluían tareas de investigación y formación de investigadores, la mayor parte orientadas hacia áreas como la física, la matemática, la radiación cósmica, la astronomía, la biología, la botánica y la genética.

El primer instituto en organizarse fue el Instituto de Aerofísica, Radiación Cósmica y Meteorología, creado en noviembre de 1950.54 Para dirigirlo, la universidad puso al frente a Walter Georgii, quien en junio de ese año dictó una serie de conferencias acerca de las condiciones de la atmósfera y el aire en la provincia para el vuelo de planeadores. bc En el instituto trabajó también Guillermo Bibl. ${ }^{55}$

Georgii representó a la universidad en diversos eventos, conferencias e instituciones sobre vuelo a vela, tales como la Sociedad Científica Aeronáutica de Brasil, el Congreso de la Organización Científica y Técnica Internacional de Vuelo a Vela, realizado en Madrid en mayo de $1952 .{ }^{56}$

Entre las investigaciones efectuadas por Georgii, se encuentran varios estudios sobre el polvo atmosférico de la Argentina y Mendoza, así como experimentos de producción de lluvia artificial con siembra de hielo sobre las sierras de San Luis. ${ }^{57}$ Georgii también colaboró con Arturo Corte y Wolfgang Ehrenberg en investigaciones sobre el aprovechamiento de la radiación solar para usos agrarios. La idea básica de este trabajo era la utilización de polvo con coloración blanca (utilizando ceniza volcánica) o negra (empleando hollín negro o residuos de combustión incompleta de aceites) para una menor o mayor absorción de los rayos solares, como técnica de regulación del ciclo de riego y su aprovechamiento en las plantaciones. ${ }^{58}$ En el mismo sentido se concretó la visita del doctor Nathan Robinson de la Universidad de Haifa (Israel) para hablar sobre las aplicaciones de la radiación solar. ${ }^{59}$

Otras actividades destacables de esta sección fueron las investigaciones realizadas en el campo de la radiación cósmica en torno a la Estación de Altura. Los trabajos comenzaron bajo la dirección de Pinardi a mediados de 1948, con la implementación del método de exposición de placas nucleares. El proyecto de instalación del Observatorio de Rayos Cósmicos orientó la indagación hacia el estudio de la radiación libre proveniente del espacio. La iniciativa del italiano, sirvió como base para las tareas realizadas en las instalaciones de Laguna Diamante, por un grupo de profesores y estudiantes de la universidad de Buenos Aires, constituido por Hans Kobrak, Beatriz Cougnet, Pedro Waloschek y Juan Roederer. Las exposiciones de placas nucleares efectuadas por el grupo en el observatorio de la universidad cuyana, posibilitaron publicaciones internacionales y temas para doctorado. Estas experiencias iniciadas por Pinardi y 
continuadas por el grupo de la Facultad de Ciencias de Buenos Aires, constituyen antecedentes importantes para la historia de la astronomía de altas energías en la provincia y la región cuyana. ${ }^{61}$

En este sentido, los trabajos de colaboración de la universidad mendocina y del ejército, poniendo a disposición la logística, las instalaciones y parte de su personal, fueron centrales en la consolidación de los estudios sobre radiación cósmica, señalando una orientación futura de la casa de estudios en este campo.

El Instituto de Matemática comenzó a funcionar sobre la base de algunos integrantes del Centro de Estudios Físico Matemáticos, entre los que se destacó Antonio Monteiro ${ }^{62}$ como miembro del futuro instituto. En 1950 se propuso un proyecto que sentaba los pilares organizativos del Instituto de Matemática, planteándose como objetivos la investigación y formación de investigadores en el campo de la matemática, la edición de publicaciones especializadas, la organización de una biblioteca, así como la promoción de intercambios con otras instituciones científicas y con los demás institutos del Departamento. ${ }^{63}$ La actividad se intensificó a partir de 1953, con la contratación de Mischa Cotlar como director del instituto, y de varios profesores que conformaron un grupo reconocido internacionalmente. El mismo estuvo integrado por mismo Monteiro, Rodolfo Ricabarra, Oscar Varsavsky, Orlando Villamayor, Dietrich Voelker, Eduardo Zarantonello, Gregorio Klimovsky y Jorge Bosch. Como becarios se incorporaron al Instituto de Matemática los estudiantes Enzo Gentile, que obtuvo su título de licenciado allí, Rafael Panzone (1956), que por recomendación de Yanny Frenkel completaba algunos seminarios para su licenciatura en Matemática de la Facultad de Ciencias Exactas y Naturales de la Universidad de Buenos Aires, Pericles Guaycochea y Lissetta Bruschi. ${ }^{64}$

Este Instituto organizó una importante biblioteca, tal como estaba previsto en sus bases fundacionales. El material se fue acrecentando con compras de libros y revistas especializadas, las que llegaron a constituir un fondo bibliográfico de aproximadamente tres mil ejemplares, posiblemente el mejor de la época y cuyo destino fue en parte San Luis. ${ }^{65}$

Entre las actividades relevantes organizadas por miembros del Instituto de Matemática, se encuentran en colaboración con la Unión Matemática Argentina, las "Sextas Jornadas Matemáticas Argentinas" realizadas en San Luis en septiembre de 1953, y las "VIII Jornadas Matemáticas" en $1955 .{ }^{66}$

En conjunto con la Unesco el instituto organizó el "Segundo Symposium Matemático sobre algunos problemas que se están estudiando en América Latina", realizado en julio de 1954, los "Primeros Cursos Latinoamericanos de perfeccionamiento en Matemáticas para Profesores Universitarios", efectuados entre febrero y marzo de 1955 en Mendoza, así como la visita del matemático francés Arnaud Denjoy. ${ }^{67}$

Asimismo, a principios de 1956, los investigadores de la sección recibieron una delegación peruana. Permanecieron en Mendoza durante cuatro semanas para asistir a los cursos dictados por los miembros del organismo mendocino. ${ }^{68}$

La repercusión de las actividades del Instituto de Matemática del DIC señaló una etapa fundamental en la historia de esa disciplina en nuestro país, con ampliaciones en el ámbito internacional y latinoamericano ${ }^{69}$ Julio Rey Pastor, Jorge Bosch y Rafael Panzone, han reconocido la importante contribución del Instituto de Matemática del DIC. ${ }^{70}$

En el seno del Departamento de Investigaciones Científicas existió también un Instituto de Física, posteriormente denominado Instituto de Física Nuclear. El mismo figuraba en los planes desde fines de 1949, pero se concretó en junio de $1950^{71}$ y fue una base para posteriores vinculaciones entre la universidad, la Comisión Nacional de Energía Atómica y la creación del Instituto Balseiro en 1955.

Las actividades sobre física nuclear tuvieron un fuerte impulso en el país y en la universidad cuyana debido al contexto internacional. El Instituto de Física Nuclear del DIC, tuvo a su cargo toda la actividad vinculada a la energía nuclear en la región pero con proyecciones nacionales. En 1951 se firmó un acuerdo que duró hasta 1954, entre la universidad y la CNEA, para que la casa de estudios colaborara en los programas de investigación nuclear de la Comisión. En ese marco, uno de los protagonistas más representativos en el Departamento fue el ingeniero Otto Gamba, quien realizó intensas gestiones para consolidar el campo disciplinar e intervino en la mayoría de las acciones. Un acontecimiento relevante fue la compra de un sincro-ciclotrón a Holanda diseñado por Claus Bakker, y que la universidad realizó 
gracias a la mediación de Gamba. ${ }^{72}$ Si bien fue comprado oficialmente por la universidad cuyana, el aparato se instaló en laboratorios de la CNEA en Avenida del Libertador de Buenos Aires. ${ }^{73}$

Una actividad relevante fue la visita a Mendoza de físicos europeos a fines de 1950. Las actividades vinculadas con las aplicaciones de radioisótopos a la salud, ocuparon asimismo un lugar importante.

Entre junio y julio de 1954, el Instituto de Física Nuclear organizó cursos para posgraduados sobre reactores nucleares, a cargo de Agustín Durañona y Vedia, Guillermo Bibl, Fausto Toranzos y Dietrich Voelker. ${ }^{74}$

Por otra parte, las actividades de exploración y explotación de mineral de uranio que venía realizando personal del Departamento de Combustibles de la universidad, fueron promovidas bajo la órbita del Dic, durante la dirección de Georgii y la vicedirección de Gamba. ${ }^{75}$

De esta manera, la colaboración universidad (DIC)-CNEA constituyó el germen que dio sustento a la conformación de las estructuras con que la entidad nacional organizó posteriormente los trabajos sobre el uranio en el país. ${ }^{76}$

El DIC tuvo también una sección Biología, dividida a su vez en sección o Instituto de Biología Animal, integrada por Armando Pisanó y José M. Cei, y sección o Instituto de Biología Vegetal, compuesto por Adrián Ruiz Leal, Heinz Brücher y Fidel Roig. ${ }^{77}$

Ambas secciones organizaron expediciones al norte del país para recolectar especies animales y vegetales para su estudio en el Departamento. ${ }^{78}$

Una mención aparte lo constituye el genetista Enrique (Heinz) Brücher, que desarrolló una intensa actividad de investigación y divulgación sobre genes de resistencia de las plantas cultivadas. Integró varias expediciones científicas de recolección de especies, organizó una conferencia, la visita del botánico Otto Stocker, y una muestra en el laboratorio del DIC de las colecciones obtenidas. ${ }^{79}$

Existieron intentos de conformar otras áreas o secciones en el Departamento. A mediados de 1950, se dictaron cursos de radio, con el objetivo de organizar la carrera de ingeniero electrónico ${ }^{80}$

Entre las iniciativas de investigación del DIC orientadas a problemas económicos, se destaca el intento de elaborar en septiembre de 1950 un plan de investigaciones y exploraciones en alta montaña con el objetivo de estudiar la importancia de los deshielos cordilleranos para la riqueza vegetal y energética de la región. Además, puede mencionarse la colaboración de la Universidad de Cuyo y la CNEA en 1951, para estudiar las posibilidades de aplicación de los métodos y técnicas de la física nuclear al problema de la filoxera ${ }^{81}$ que afectaba fuertemente a la industria vitivinícola. ${ }^{82}$

La formación de recursos humanos se organizó mediante la participación de los profesores en eventos científicos y la convocatoria a científicos nacionales y extranjeros, principalmente europeos, para dictar conferencias sobre temas astronómicos, de física nuclear, biológicos, médicos y matemáticos. ${ }^{83}$ También mediante la residencia de delegaciones extranjeras en la universidad para formarse con investigadores del departamento. Asimismo, la formación de becarios era uno de los objetivos del organismo, pero solamente en la sección de matemáticas se pudo confirmar la existencia de estudiantes becados y un estudiante no identificado que publicó una reseña de actividades del organismo en un periódico estudiantil de $1951 .{ }^{84}$

El DIC editó algunas publicaciones periódicas especializadas, ${ }^{85}$ como los Anales del Departamento de Investigaciones Científicas, boletín con las publicaciones de varias secciones del organismo y una duración de seis años (1952-1957), ${ }^{86}$ y la Revista Matemática Cuyana (1955-1958), a cargo del Instituto de Matemática y con una existencia de tres años. ${ }^{87}$ En esta publicación, además de contribuir con sus trabajos los integrantes del Instituto de Matemática o vinculados a este (Villamayor, Cotlar, Sispánov, Ricabarra, Zarantonello, Monteiro, Varsavsky), uno de los fascículos estuvo compuesto por la colaboración de los investigadores extranjeros, Norbert Wiener y Aurel Wintner, del Instituto Tecnológico de Massachussets y de la Universidad John Hopkins (Estados Unidos) respectivamente, y Whilhelm Blaschke (Hamburgo). ${ }^{88}$ 
La dimensión política del DIC constituyó en la práctica una excepción respecto a la situación de persecución y cesantías de profesores que vivían las universidades del país, incluyendo la propia universidad cuyana. El protagonismo del rector Cruz y su estrecha vinculación con el presidente, posibilitaron un ambiente de trabajo en el organismo científico, relativamente libre de condicionamientos políticos, situación que se mantuvo incluso después de la muerte del rector hasta 1955. El ejemplo más claro fue el Instituto de Matemática, donde se logró una coexistencia de diversas ideologías, incluso contrarias al régimen. Al respecto, el profesor Bosch ilustra bien el contexto que se vivía:

En aquella época, para tener acceso a empleos del Estado era necesario estar afiliado al Partido Peronista y participar en los actos de pleitesía propios del régimen. El Dr. Cruz le planteó a Perón que con esas condiciones era imposible reclutar gente de alto nivel para la creación científica pura y que, en consecuencia, el Instituto de Matemática debía estar exento de tota exigencia política. Perón aceptó y entonces el rector Cruz, con el asesoramiento del Dr. Monteiro, convocó al Dr. Misha Cotlar para que formara y dirigiera un equipo de matemáticos que trabajarían en las condiciones descritas. Misha Cotlar propuso entonces a Eduardo Zarantonello, Rodolfo Ricabarra, Oscar Varsavsky, Orlando Villamayor, Gregorio Klimovsky y a mí, todos los cuales éramos antiperonistas, pero no se nos preguntó nada acerca de nuestra orientación política. Así fue creado el Instituto de Matemática en el seno del DIC en la ciudad de Mendoza, con hermosas instalaciones en Chacras de Coria. Antes de mi llegada a Mendoza el rector Cruz murió y la Universidad de Cuyo quedó en manos de su equipo, que era un conjunto de profesores nacionalistas peronistas que compartían las ideas de Cruz y que respetaron escrupulosamente lo pactado con Monteiro y Cotlar. Monteiro permaneció en San Juan, sin tener vinculación formal con el Instituto pero continuó siendo un ferviente colaborador y un eficaz apoyo en todo momento. En el DIC, en general, había amplia libertad académica y en ningún momento advertí presión alguna de carácter ideológico o político, lo cual contrastaba fuertemente con el clima que se vivía en las facultades, inclusive en las de la Universidad de Cuyo, en las cuales regía un absoluto acatamiento al peronismo. ${ }^{89}$

En el mismo sentido, otras opiniones caracterizan el clima político y de trabajo: "Recuerdo que reinaba dentro del DIC un fuerte espíritu de trabajo. No recuerdo hubiera ningún tipo de planteo ideológico o político condicionante de la labor, por parte de las autoridades". ${ }^{90}$

A partir de 1955 el Consejo Técnico del DIC integrado por Miguel Marzo, Cotlar, Cei, Cesco y Juan Carlos Fasciolo elaboró un proyecto de reestructuración. En ocasión de los cursos para profesores latinoamericanos, Marzo anuncia que el organismo se denominaría "Departamento de Investigaciones Científicas Tecnológicas". Esa reforma se orientaba a una adecuación del organismo científico al segundo Plan Quinquenal. ${ }^{91}$ Con todo, todavía se mantenía vigente la finalidad originalmente planteada para el Departamento, a la vez que se tomaba conciencia de la magnitud del proyecto. En el mismo evento en que Marzo anuncia el cambio de denominación, Oscar Dodera Luscher, representante de la Unesco, sostuvo: "el problema de la investigación científica es decisivo para el desarrollo científico de un país. Esta Universidad de Cuyo ha comenzado ya su solución y ella es la organización del Departamento de Investigaciones Científicas". ${ }^{22}$

Con la creación del DIC se fortalecieron las relaciones del gobierno universitario con el Ministerio de Asuntos Técnicos, del que dependía la actividad científica. Desde la universidad mendocina surgió el planteo ante el gobierno de Juan Domingo Perón, de conformar un organismo nacional que centralizara y coordinara la política de investigación científica y tecnológica. En el discurso de 1949, donde propuso el proyecto del DIC, el rector Cruz sostenía: "será cuestión de que nosotros lleguemos a demostrar la necesidad, de una vez por todas, y de una manera enérgica, de crear esa gran casa de estudios superiores de investigación. Hay que coordinar esfuerzos para llegar a esta realidad. Llevar a la conciencia nacional y por tanto al gobierno de La Nación la urgencia de instaurar ese gran centro nacional de investigaciones científicas". ${ }^{93}$

La urgencia señalad por el rector se concretó primero al crearse la Dirección Nacional de Investigaciones Técnicas (DNIT), ${ }^{94}$ y posteriormente cuando el gobierno instituye bajo dependencia del Ministerio de Asuntos Técnicos, el Consejo Nacional de Investigaciones Técnicas y Científicas (CONITYC) ${ }^{95}$ primer organismo científico del país, ocho años antes que el Consejo Nacional de Investigaciones Científicas y Técnicas (CONICET). El rector Cruz formó parte de 
este Consejo y realizó gestiones para promover la carrera de investigador científico en el país. En septiembre de 1951, recién conformado el CONITYC, Cruz viaja a Buenos Aires a una reunión de ese órgano. La prensa local informó del viaje y señaló: “El doctor Cruz presentará una ponencia referida a la creación de la Carrera de Investigación Científica” ${ }^{96}$ En la articulación con el ministerio mencionado, colaboró también el Secretario General de la universidad, el periodista Enrique Oliva. ${ }^{97}$ No obstante, esos intentos de promover una política científica regional y nacional por parte de los funcionarios universitarios fueron truncados por el golpe de 1955.

\section{El golpe militar de 1955 y la disolución del DIC}

En el contexto del golpe militar de 1955, las universidades constituían para los dictadores elementos de oposición cultural y política a la implementación de las medidas adoptadas, y obstáculos que debían ser removidos para eliminar todo rastro de peronismo. En tal sentido, en las universidades del país se designaron interventores que respondían al régimen militar. Toda iniciativa emprendida por el gobierno peronista (1945-1955) comenzó sistemáticamente a ser desarticulada.

En nuestra región cuyana el interventor nombrado en la Universidad Nacional de Cuyo fue Germinal Basso, doctor de profesión, había sido encarcelado por el gobierno peronista, lo que lo condujo a posiciones fuertemente antiperonistas. Puso a su vez en funciones a diversos interventores en cada una de las dependencias universitarias, incluyendo el Departamento de Investigaciones Científicas.

Las reformas funcionales implicaban cambios radicales en la política científica y en un organismo de investigación científica que hasta ese momento había tenido cierta continuidad en sus actividades, y algunas de sus secciones habían logrado trayectoria y reconocimiento. Para ello, era necesario proceder con precaución y establecer mecanismos de decisión concretos, que aunque fueran cuestionables y sus objetivos iniciales no se correspondieran con las medidas adoptadas, debían ofrecer una imagen de legitimidad democrática en las acciones. Con ello, el relativo equilibrio político-ideológico logrado en el marco del DIC durante el gobierno de Cruz, se vio afectado en el núcleo mismo de las posibilidades de establecer acuerdos.

A comienzos de 1956, la intervención universitaria conformó una comisión que debía ofrecer una nueva organización del funcionamiento administrativo del DIC. ${ }^{98}$ La comisión estuvo integrada por Adrián Ruiz Leal, Dante Nesossi, Enrique Brücher, Armando Pisanó, Mischa Cotlar, Andrés Hejjas, Guillermo Bibl, además de tres representantes de la intervención. Los debates en el seno de la misma giraron en torno a la finalidad de las investigaciones y actividades del departamento. Se plantearon claras divergencias entre los objetivos propuestos por los funcionarios oficiales, José Luis Minoprio, investigador e interventor del organismo, ${ }^{99}$ Antonio López Aguado y Raúl Slukich Daniele, ambos pertenecientes a la Comisión Investigadora de Actividades Administrativas de la universidad, y los miembros del Instituto.

En abril de ese año, dos integrantes del DIC publicaron un artículo en una revista científica nacional, alertando sobre las diferencias y los intereses puestos en juego en las discusiones mantenidas. Los interventores plantearon el debate en términos de "ciencia dirigida", mientras que varios miembros del departamento sostenían la "ciencia pura" como modalidad con la que originariamente fue concebido el organismo. Los autores de la nota, con expresiones que recuerdan el discurso de Cruz en 1949, concluían:

\footnotetext{
Habiendo sido fundado el Departamento de Investigaciones Científicas expresamente para la investigación pura, posiblemente el único caso en el país de un instituto universitario con esta sola finalidad, y que por otra parte ha dado muestras de una labor eficiente, especialmente dentro del grupo de los matemáticos que data de mayor antigüedad, consideramos que debe respetarse su finalidad y darle el respaldo ampliando sus posibilidades. Lamentamos que aún la "ciencia dirigida" se levante como una amenaza contra el investigador y esperamos que el Departamento de Investigaciones Científicas no salga del cauce en que fuera encarrilado. ${ }^{100}$
} 
Otros investigadores del DIC han señalado también la existencia de factores ideológicos o políticos, así como la persecución directa sobre varios de los integrantes por su ascendencia judía, principalmente pertenecientes al grupo de matemáticos. ${ }^{101}$ Estas persecuciones responderían a factores de orden religioso, puestos en juego por grupos de presión vinculados a facciones de derecha y antiperonistas de la Iglesia. Es un aspecto que el autor no ha podido profundizar, aunque al parecer habrían predominado razones más de orden ideológico y político que religioso en las discusiones que desencadenaron el cierre del Departamento.

Con todo, ni la intensa actividad desarrollada ni la importancia a nivel latinoamericano de los eventos realizados, como tampoco el reconocimiento y las proyecciones internacionales de los investigadores nucleados alrededor del Instituto de Matemática, fueron suficientes para evitar que la intervención disolviera el Departamento, mediante una disposición fechada el 31 de octubre de 1957 y publicada por la prensa local tres días después. ${ }^{102}$ Los motivos para disolver el organismo se centraron en tres argumentos: a) una finalidad no justificada en la práctica en relación al presupuesto universitario, b) una superposición con la tarea de investigación realizada por cada facultad, y c) una falla de su estructura consistente en la restricción en el aporte de alumnos y la formación de discípulos. Se reconoce la jerarquía del Instituto de Matemática, pero se afirma que no es posible reconstituir el equipo de trabajo, cuyos miembros para entonces habían concretado el éxodo hacia otras universidades. ${ }^{103}$ El personal, equipamiento e infraestructura se distribuyeron entre las diferentes facultades y dependencias universitarias. El observatorio "Félix Aguilar" pasó a la órbita de la Facultad de Ingeniería en San Juan, mientras que sobre los observatorios de Laguna Diamante y Cerro Pelado la ordenanza no especificaba nada. Finalmente, predominaron los intereses de la intervención y el Departamento de Investigaciones Científicas fue disuelto. A pesar de la denuncia publicada en la revista Ciencia e Investigación, no se ha podido establecer si existieron y cuáles fueron las reacciones de la comunidad científica local por el desmantelamiento.

\section{Consideraciones finales}

La creación y puesta en funcionamiento del Departamento constituyó "uno de los intentos más audaces e importantes que se hayan hecho en materia científica y tecnológica"104 y su disolución fue una "pérdida irreparable". ${ }^{105}$

La organización del DIC implicó, por una parte, definir un programa de grado y posgrado destinado a la formación de recursos humanos y a la organización de la carrera de investigador. Por otra, una orientación hacia la perspectiva de conformar un organismo nacional que concentrara la investigación científica. En este sentido, el proyecto constituye un antecedente institucional relevante en la conformación del complejo científico y tecnológico.

De este modo, el DIC significó la puesta en práctica de una estrategia para impulsar una política científica a escala regional en Cuyo, y al mismo tiempo nacional. El Departamento representó una modalidad de institucionalización de la ciencia, enmarcada en una política de transferencia científica y tecnológica, y un modelo institucionalizador basado preponderantemente en el "factor humano", donde el elemento decisivo fue la presencia de científicos formados con los medios adecuados de trabajo. ${ }^{106}$ En consecuencia, la iniciativa suponía una considerable coordinación de esfuerzos como requerimiento para lograr los objetivos propuestos. Ello implicó sujetar la estructura del organismo científico y sus actividades, a las exigencias del complejo contexto político internacional de posguerra y del modelo de desarrollo económico adoptado, tendencias expresadas en la política de transferencia científico-tecnológica y en la necesidad de industrialización.

A lo largo de sus ocho años de permanencia en el tiempo, participaron en el Departamento docentes e investigadores vinculados a la universidad local, pero también se incorporó una apreciable cantidad de recursos del exterior, principalmente de países europeos derrotados en la guerra, como Italia, y en mayor medida Alemania. Con todo, la iniciativa evidencia características particulares que no pueden reducirse exclusivamente a la mera recepción de modelos organizativos o institucionales extranjeros. En el caso de la sección matemática por ejemplo, sus integrantes no se limitaron a repetir simplemente desarrollos teóricos producidos a nivel internacional, sino que a partir de esos desarrollos 
generaron nuevos problemas de investigación. Por su parte, la sección de biología animal, carecía de las condiciones suficientes para trabajos de laboratorio, lo que evidencia particularidades de un contexto periférico de investigación.

La mayor parte de las secciones del organismo no sobrevivieron a la existencia de éste, salvo aquellas que tenían un interés económico para los militares golpistas, tales como los estudios sobre papas de la sección de botánica y biología vegetal, y los trabajos de explotación de minerales de uranio continuados en el marco de la CNEA.

La institución tuvo importante presencia en el medio cultural mendocino, con diversas actividades de investigación y formación de investigadores, fundamentalmente en las áreas de energía nuclear, matemática y biología El golpe de 1955 desmanteló el organismo científico universitario. Con ello, la disolución del DIc significó que las posibilidades de continuar, reorientar o profundizar la política científica y las estrategias de institucionalización de la ciencia iniciadas entre 1948 y 1950 en la provincia y la región cuyana, fueron desarticuladas.

EI DIC fue el comienzo de una incipiente cristalización y consolidación de disciplinas y áreas como la meteorología, la física nuclear, la matemática, la biología, la botánica y la medicina. Su impronta puede apreciarse en formas de investigación y enseñanza que aun se mantienen en la universidad cuyana. En este sentido, el desarrollo de la investigación científica se continuó sobre la base de la conexión lineal referida entre ciencia básica, ciencia aplicada, técnica e industria, lo que probablemente haya dificultado una mayor incidencia del organismo en la actividad científica nacional. Las actividades del DIC promovieron una cultura científica en la provincia y la región entendida bajo ese enfoque lineal.

No obstante, algunas consecuencias pueden apreciarse en la formación y en las posibilidades de investigación de sus integrantes. Varios de ellos se constituyeron en los años posteriores en formadores de estudiantes y ocuparon cargos en diversas instituciones científicas y académicas del país.

En conjunto, estos aspectos resultan claves para la posibilidad de comprender adecuadamente y en su justa medida, la relevancia del Departamento de Investigaciones Científicas en el proceso de institucionalización de la ciencia en Mendoza y Cuyo.

\section{Notas e referências bibliográficas}

Pablo Antonio Pacheco é professor em Filosofia (Universidad Nacional de Cuyo (UNCuyo). Licenciado em Filosofia (orientação em Epistemologia e História da Ciência). Doutorando em Ciências Sociais (Faculdade de Ciências Políticas e Sociais, UNCuyo). Membro do Programa de Investigaciones sobre Dependencia Académica en América Latina (PIDAAL), liderada pelo Doutora Fernanda Beigel (CONICET-UNCuyo).E-mail: pablopach@hotmail.com.

1 El presente trabajo constituye un avance de resultados del proyecto "El Departamento de Investigaciones Científicas de la Universidad Nacional de Cuyo y las actividades científicas en la región entre 1949 y 1957" (Etapas I y II) que el autor ha realizado en las convocatorias 2008-2009 y $2009-2010$ y del proyecto "El contexto institucional de la ciencia en Mendoza y Cuyo (1948-1957)" que desarrolla como becario de la Secretaría de Ciencia, Técnica y Posgrado de la UNCuyo (convocatoria 2010-2011), en el marco del proyecto "Los contextos de la ciencia", que dirige el profesor Guillermo Alberto Cuadrado. Actualmente, los problemas de investigación presentados en el artículo han sido presentados para su consideración como proyecto de posgrado en el doctorado en Ciencias sociales (Mención sociología) de la Facultad de Ciencias Políticas y Sociales (UNCuyo) que coordina la Dra. Fernanda Beigel. El autor reconoce la amabilidad de aquellas personas dentro y fuera de la universidad mendocina que facilitaron el acceso a fuentes, aportaron información complementaria, aceptaron ser entrevistadas o contribuyeron con sus testimonios. Asimismo, agradece especialmente al doctor Eduardo L. Ortiz del Imperial College (Londres) por sus comentarios críticos.

2 Véase MANTEGARI, Cristina. Museos y ciencias: algunas cuestiones historiográficas. In: MONSERRAT, Marcelo (Comp.). La ciencia en la Argentina entre siglos: textos, contextos e instituciones. Buenos Aires: Manantial, 2000. p. 297-308 y VESSURI, Hebe. 0 inventamos o erramos: La ciencia como idea-fuerza en América Latina. Buenos Aires: Editorial de la Universidad Nacional de Quilmes, 2007. p. 185.

3 Véase HURTADO DE MENDOZA, Diego; BUSALA, Analía. De la "movilización industrial" a la Argentina científica: la organización de la Ciencia durante el peronismo (1946-1955).Revista da Sociedade Brasileira de História da Ciência, Rio de Janeiro, Sociedade Brasileira de História da Ciência, v. 4, n. 1, p. 18, 2006.

4 Existen trabajos que desde diversas perspectivas, abordan aspectos relevantes de la ciencia y la técnica durante el período. Entre ellos, pueden mencionarse a HYMANS, Jacques. The psychology of nuclear proliferation: identity, emotions and foreign policy. New York: Cambridge Universty Press, 2006 (con un capítulo sobre el caso Richter); sobre el diseño de los aviones Pulqui I y II véase LALOUF, Alberto. Un modelo tentativo para el análisis de la producción de artefactos tecnológicos en países subdesarrollados: más allá de la fracasomanía. In: KREIMER, Pablo; THOMAS, Hernán; ROSSINI, Patricia; LALOUF, Alberto (Eds.). Producción y uso social de conocimientos: estudios de Sociología de la Ciencia y la tecnología en América Latina. Buenos Aires: Editorial de la Universidad Nacional de Quilmes, 2004. p. 263-286, así como los trabajos de Mariscotti y Myers mencionados en este artículo. 

peronismo (1946-1955). Revista da Sociedade Brasileira de História da Ciência, Rio de Janeiro, Sociedade Brasileira de História da Ciencia v. 4, n. 1, p. 24-25, 2006; MYERS, Jorge. Antecedentes de la conformación del Complejo Científico y Tecnológico, 1850-1958. In: OTEIZA, Enrique (Dir.). La política de investigación científica y tecnológica argentina. Historia y perspectivas. Buenos Aires: Centro Editor de América Latina, 1992. p. 100 y ORTIZ, Eduardo L.; RUBINSTEIN, Héctor. La Física en la Argentina en los dos primeros tercios del siglo veinte: algunos condicionantes exteriores a su desarrollo. Revista Brasileira de História da Ciencia, Rio de Janeiro, Sociedade Brasileira de História da Ciencia, v. 2, n. ${ }^{0}$ 1, p. 43, 2009,

6 MYERS, op. cit., p. 100

$7 \quad$ Véase MYERS, op. cit., p. 98-100.

8 Cf. VILAS, Carlos María. La dominación imperialista en Argentina. Buenos Aires: Eudeba, 1974. p. 39-78 y CARDOSO, Fernando Enrique; FALETTO, Enzo. Dependencia y desarrollo en América Latina. Ensayo de interpretación sociológica. Buenos Aires: Siglo xxI, 2005. p. 102-129.

9 Véase SÁNCHEZ CRESPO, Alberto. Esbozo del desarrollo industrial de América latina y de sus principales implicaciones sobre el sistema científico y tecnológico. In: SUÁREZ, Francisco; CIAPUSCIO, Héctor; KRIEGER, Mario; AMADEO, Eduardo (Comps.). Autonomía nacional o dependencia: la política científico-tecnológica. Buenos Aires: Paidós, 1975. p. 66-113.

10 Cf. HURTADO DE MENDOZA; BUSALA, op. cit.

11 Cf. OTEIZA, Enrique (Dir.). La política de investigación cientifica y tecnológica argentina. Historia y perspectivas. Buenos Aires: Centro Editor de América Latina, 1992. p. 21.

12 Cf. COMASTRI, Hernán. Científicos alemanes en la Argentina peronista. Límites y potencialidades de una política de transferencia científico-tecnológica. Antíteses, Brasil, v. 2, n. 4, jul.-dez. de 2009. p. 693-710. Disponível em: <http://www.uel.br/revistas/uel/index.php/antiteses>. Acceso em: 23 mar. 2010. El autor agradece al doctor Dante Ramaglia del Centro Científico y Tecnológico (CCT-Mendoza) por la sugerencia de este artículo.

13 Cf. ORTIZ; RUBINSTEIN, op. cit., p. 64-70; HURTADO DE MENDOZA, Diego; BUSALA, Analía. Los ideales de universidad "científica" (1931-1959): elitismo y función social de la ciencia en la Argentina. Buenos Aires: Libros del Rojas, 2002.

14 VESSURI, Hebe, op. cit., p. 191-195, señala que en la definición de la agenda de investigación en el contexto de una modernización (1940-1970), el patrón de industrialización sustitutiva adoptado, condujo a que las élites nacionales, con ayuda de las agencias internacionales, transmitieran a los gobiernos latinoamericanos la visión de un flujo lineal, unidireccional que iba desde la investigación básica ("ciencia pura"), pasando por el desarrollo tecnológico hasta su aplicación comercial o industrial.

15 Véase por ejemplo el discurso del rector Ireneo Cruz en UNIVERSIDAD NACIONAL DE CUYO. La investigación en la Universidad. Mendoza, 1950, p. 13.

16 Cf. SÁNCHEZ CRESPO, op. cit., p. 99-100

17 Véanse Ord. nº 147/49. En UNIVERSIDAD NACIONAL DE CUYO. La investigación en la Universidad. Mendoza, 1950, p. 25 y Ord. n² 29/51. También diario Los Andes, 17/2/1950, p. 4.

18 Véase HURTADO DE MENDOZA; BUSALA, Analía, op. cit., p.24

19 Este apartado se basa en un trabajo previo elaborado por el autor. Véase PACHECO, Pablo Antonio. Estrategias de institucionalización científica en Mendoza y Cuyo (1948-1950). In: IV CONGRESO INTEROCEÁNICO DE ESTUDIOS LATINOAMERICANOS. Actas.... Mendoza, 2010.

20 Cf. UNIVERSIDAD NACIONAL DE CUYO. Memoria de la intervención. 1946-1947. Mendoza: Ministerio de Justicia e Instrucción Pública, Universidad Nacional de Cuyo, 1947. p. 71-75.

21 Otto Gamba tuvo una activa e importante participación en los estudios de física nuclear en el país. Colaboró en la comisión encargada de evaluar el Proyecto Huemul, así como en reclutar a un grupo joven para trabajar en la recién creada Comisión Nacional de Energía Atómica a principios de la década de 1950, organismo del cual fue también funcionario. Véase LÓPEZ DÁVALOS, Arturo; BADINO, Norma. J. A. Balseiro: crónica de una ilusión. Una historia de la Física en la Argentina. Buenos Aires: Fondo de Cultura Económica, 2000. p. 161-164.

22 Véase Res. $n^{0}$ 36/48, 47/48 y 120/49, Universidad Nacional de Cuyo, Archivo Oficina General de Despacho (AOGD-UNCuyo). Véase también Los Andes, 9/12/48, p. 4; 25/12/49, p. 6; 26/12/49, p. 4 y 27/12/49, p. 2-3. Sobre la creación del Museo Tecnológico y Laboratorios de Investigación en la Universidad Nacional de Cuyo cf. PACHECO, Pablo Antonio. Creación del Museo Tecnológico y Laboratorios de Investigación de la Escuela de Ingeniería en Combustibles (UNCuyo). In: GÓMEZ, Luis; CUADRADO, Guillermo (Ed.). Educación de ciencias empíricas en carreras de ingeniería. Mendoza: Universidad Tecnológica Nacional, Facultad Regional Mendoza, 2010. p. 105-114. La conexión con la iniciativa desarrollada por Gustavo Fester en la Universidad Nacional del Litoral fue establecida gracias a la lectura de MATHARAN, Gabriel Augusto. La emergencia e institucionalización de la Química como disciplina en la ciudad de Santa Fe (1911-1935). In: PREGO, Carlos A.; VALLEJOS, Oscar (Comp.). La construcción de la ciencia académica: instituciones, procesos y actores en la universidad argentina del siglo XX. Buenos Aires: Biblos, 2010, p. 79-103.

23 Entre sus veintisiete miembros, se encontraban Carlos Ulrico Cesco, Bernard H. Dawson, Modesto González, Fausto Ismael Toranzos, Enrique Loedel Palumbo, Pascual Colavita, Enzo 0. Macagno, Pedro Pi Calleja, Giovanni Pinardi, Sergio Sispanov, César Trejo, Pedro Gerardo Cabezas, Fernando Volponi, Orlando Villamayor, Carlos Corti Videla, Manuel Balanzat, y Juan J. Nissen. Varios de ellos fueron activos colaboradores de instituciones científicas de sus especialidades, como la Unión Matemática Argentina (UMA) y La Asociación Física Argentina (AfA).

24 UNIVERSIDAD NACIONAL DE CUYO. Actas del Primer Congreso Nacional de Filosofía. Mendoza, 1950. tomo III, p. 1.589-1.639.

25 Los Andes, 21/4/1948, p. 6; 3/5/1948, p. 10; 6/5/1948, p. 6 y 2/6/1948, p. 7.

26 Véase ASOCIACIÓN CIENTíFICA DE MENDOZA. Boletín informativo. Mendoza, v. I, n. I, agosto 1949.

27 Mediante la Ord. n 122, del 4 de octubre de 1949.

28 Véase Res. № 267, del 17 de marzo de 1948, el Decreto nº 23.471, del 22 de septiembre de 1949 y el Expediente $n^{0}$ 10.653-M (Legajo n 1.785). (AOGD y Archivo Oficina de Recursos Humanos-UNCuyo.)

29 Cf. el proyecto presentado por Pinardi Observaciones acerca de los aspectos económicos de la creación de un Centro de Física Nuclear en la Universidad Nac./Cuyo, Mendoza, septiembre de 1948 (gentileza de Giovanni Pinardi). Para estos y otros detalles de las actividades del italiano en torno al proyecto del Observatorio de Rayos Cósmicos de la Universidad Nacional de Cuyo, véase PACHECO, Pablo Antonio. La construcción del Observatorio de Rayos Cósmicos 
de la Universidad Nacional de Cuyo: el doctor Giovanni Pinardi y las primeras investigaciones sobre radiación cósmica en la región. In: $4^{0}$ ENCUENTRO INTERNACIONAL DEL ICES (E-ICES-4). Actas... Buenos Aires: CNEA..

30 UNIVERSIDAD NACIONAL DE CUYO. La investigación en la Universidad. Mendoza, 1950. p. 11. Véase también la entrevista de Antonio Vázquez a Ireneo Cruz, La Libertad, 25/12/49, p. 8.

31 La ord. nº 147/49 lo crea, la nº 9/50 establece las líneas generales de su organización y funcionamiento, la n ${ }^{0} 6 / 51$ establece los planes de estudio, la $n^{0}$ 8/51 fija sus institutos, la n ${ }^{0}$ 14/51 a pedido de alumnos homologa las carreras de la Facultad de Ingeniería y Ciencias Exactas, Físicas y Naturales con las del DIC, la n $n^{0}$ 29/51 reorganiza y asume los cambios hechos y la $n^{0}$ 54/57 finalmente lo disuelve (AOGD-UNCuyo).

32 En la mayoría de los documentos consultados se invoca reiteradamente esta ley en los incisos $2^{\circ}, 6^{\circ}$ y $12^{\circ}$ del artículo $2^{\circ}$, que establecían respectivamente entre las funciones de las universidades "Organizar la investigación científica", "Crear un cuerpo docente dedicado exclusivamente a la vida científica" y "Crear y sostener institutos de investigación, cursos de perfeccionamiento o de especialización, para profundizar el estudio 0 aprovechamiento de las riquezas naturales de la zona del país donde tuviera su centro de acción cada universidad". Véase MANGONE, Carlos; WARLEY, J. Universidad y peronismo (1946-1955). Buenos Aires: Centro Editor de América Latina, 1984. p. 95.

33 Véase por ejemplo Los Andes, 28/12/1949, p. 5; 13/2/1950, p. 6, también La Libertad, 29/12/1949, p. 5.

34 Entrevista de Antonio Vázquez a Ireneo Cruz, La Libertad, 25/12/1949, p. 8

35 UNIVERSIDAD NACIONAL DE CUYO. La investigación en la Universidad. Mendoza, 1950, p. 15.

$36 \quad$ Véase res. n 988 , del 13 de junio de 1950.

37 Cf. Ord. $n^{0} 147$, art. $4^{\circ}$ y Ord. $n^{0}$ 9, arts. $1^{\circ}-2^{\circ}$. En UNIVERSIDAD NACIONAL DE CUYO. La investigación en la Universidad. Mendoza, 1950. p. 26-31, también ord. $n^{0} 8$ de creación de institutos, del 12 de febrero de 1951, art. $1^{0}$ (AOGD-UNCuyo).

38 Cf. ord. $n^{0} 147$, art. 2. En UNIVERSIDAD NACIONAL DE CUYO. La investigación en la Universidad. Mendoza, 1950, p. 25, n. 29, art. $2^{\circ}$ (AOGD-UNCuyo).

39 Cf. las ord. n0 147/49 y 9/50. En UNIVERSIDAD NACIONAL DE CUYO. La investigación en la Universidad. Mendoza, 1950, p. 23-40, ord. nº 8/51 y 29/51, arts. $3^{\circ}-8^{\circ}$ (AOGD-UNCuyo).

40 Res. n 3.607 del 20 de diciembre de 1949 (AOGD-UNCuyo).

41 Véase LACOSTE, Pablo. La Universidad de Cuyo y sus luchas. In: LACOSTE, Pablo (Comp.). Mendoza. historia y perspectivas: aporte para el estudio de una ciudad fundada en 1561. Mendoza: Diario Uno/Universidad de Congreso, 1997. p. 142.

42 Cf. ord. nº 147/49. En UNIVERSIDAD NACIONAL DE CUYO. La investigación en la Universidad. Mendoza, 1950, p. 27.

43 Cf. ord. $n^{0}$ 29/51 (AOGD-UNCuyo).

44 Cf. ord. $n^{0} 147$, arts. $15^{\circ}$ y $22^{\circ}-25^{\circ}$. En UNIVERSIDAD NACIONAL DE CUYO. La investigación en la Universidad. Mendoza, 1950, p. 27-29 y n² 29, arts. 34$37^{\circ}$.

45 Cf. ord. n 9, art. 14. En UNIVERSIDAD NACIONAL DE CUYO. La investigación en la Universidad. Mendoza, 1950, p. 33, UNIVERSIDAD NACIONAL DE CUYO. Digesto. Guía del estudiante. Mendoza: Departamento de Ceremonial y Prensa, 1951. p. 347-350, ord. $n^{0} 6$ del 9 de febrero de 1951, y n 8 del 12 de febrero de 1951, art. $2^{\circ}$ (AOGD-UNCuyo).

46 Cf. Ord. $n^{0} 29$ del 30 de mayo de 1951, arts. $31^{\circ}$ y $33^{\circ}-35^{\circ}$ (AOGD-UNCuyo).

47 Cf. Ord. $n^{0}$ 147, art. 20º ordenanza nº 9, art. 11. En UNIVERSIDAD NACIONAL DE CUYO. La investigación en la Universidad. Mendoza, 1950, p. 28-33, n 8, art. $2^{\circ}$ (AOGD-UNCuyo). Para determinar el puntaje de los cursos se establece que a un curso de 12 semanas (tres más o menos) con tres horas semanales le corresponde un punto.

48 Cf. Ord. $n^{0}$ 147, art. 16 y n 9 9, arts. 150-19. En UNIVERSIDAD NACIONAL DE CUYO. La investigación en la Universidad. Mendoza, 1950, p. 27-28 y 33-34, n. 29, art. $26^{\circ}$ (AOGD-UNCuyo), también UNIVERSIDAD NACIONAL DE CUYO. Digesto. Guía del estudiante. Mendoza: Departamento de Ceremonial y Prensa, 1951. p. 348.

49 Cf. UNIVERSIDAD NACIONAL DE CUYO. La investigación en la Universidad. Mendoza, 1950, p. 15

50 Sobre los trabajos del Observatorio de Mendoza cf. Los Andes, 14/1/1950, p. 4; 8/3/1950, p. 4 y 1/6/1950, p. 4.

51 Véase Los Andes, 10/5/1952, p. 2; 11/5/1952, p. 4 y 12/5/1952, p. 4.

52 Véase Los Andes, 21/9/1953, p. 5; 25/9/1953, p. 3; 26/9/1953, p. 4 y días subsiguientes.

53 La primera inscripción se abre del 17 de marzo al 13 de abril de 1950, comenzando las clases el 22 de mayo. Cf. Los Andes, 17/3/1950, p. 4 y 12/4/1950, p. 6.

54 Véase ord. $n^{0}$ 63, del 15 de noviembre de 1950 (AOGD-UNCuyo).

55 Sobre estas conferencias de Georgii cf. Los Andes, 27/6/50, p. 4 y 2/7/50, p. 6.

56 Véase Los Andes, 26/1/54, p. 4.

57 Véanse res. no 827 del 31 de agosto de 1951, 449 del 16 de mayo de 1952 (AOGD-UNCuyo), también Los Andes, 22/5/1952, p. 2.

58 Cf. Los Andes, 4/1/1951, p. 4.

59 Cf. Los Andes, 12/10/1951, p. 3.

60 Véase Los Andes, 29/8/1954, p. 6, 30/8/1954, p. 4 y 1/9/1954, p. 4.

61 Cf. ROVERO, Adrián. Historia de la Astronomía de Altas Energías en Argentina. In: ROMERO, Gustavo E.; CORA, Sofía A.; CELLONE, Sergio A. (Ed.). Historia de la Astronomía Argentina. Buenos Aires: Asociación Argentina de Astronomía, 2009. p. 357-402. También ROEDERER, Juan. Las primeras investigaciones de radiación cósmica en la Argentina (1949-1959). Ciencia Hoy, Buenos Aires, v. 12, n. 71, p. 38-48, 2002; La Libertad, 5/1/1951, p. 6 y diario La Palabra, 5/1/1951, p. 4.

62 El caso de Antonio Monteiro (1907-1980) reviste especial importancia, aunque en este trabajo no hay espacio para relatar ampliamente su trayectoria. Para ello, puede consultarse The collected works of António Monteiro. 
64 Entrevista del autor al doctor Rafael Panzone (31/8/2008).

65 Entrevista del autor a los doctores Jorge Bosch (6/6/2008) y Rafael Panzone (31/8/2008).

66 Véase UNIVERSIDAD NACIONAL DE CUYO. Boletín, Mendoza, n. 6, set.-jun., p. 31-34 y Los Andes, 13/6/1955, p. 4.

67 Véase UNIVERSIDAD NACIONAL DE CUYO. Comunicaciones. Mendoza, año II, $2^{\mathrm{a}}$ quincena de marzo y $1^{\mathrm{a}}$ y $2^{\mathrm{a}}$ de abril, n. 45-47, p. 26-27. También véase UNESCO. Segundo Symposium sobre algunos Problemas Matemáticos que se están Estudiando en Latino América, Villavicencio-Mendoza 21-25 julio 1954. Montevideo: Centro de Cooperación Científica de la Unesco para América Latina, 1955, p. 6-8. El autor agradece al doctor John Horvath (Universidad de Maryland) el envío de este material. También Los Andes, 14 y 21 al 26/6/1954, 14/7/1954, p. 3; 21/7/1954, p. 4; 22/7/1954, 23/7/1954, p. 2; 24/7/1954, p. 2; 25/7/1954, p. 4 y 26/7/1954, p. 6; 3/11/1954, p. 4 y 1 , 2 y $3 / 3 / 1955$, p. 4.

68 Entrevista del autor al doctor César Carranza (29/12/2008). Véase también los Andes, 11/3/1956, p. 7.

69 Entrevista del autor al doctor César Carranza (29/12/2008).

70 Entrevista del autor a Jorge Eduardo Bosch (6/6/2008) y a Rafael Panzone (31/8/2008). Sobre la opinión de Rey Pastor véase UNESCO, op. cit., p. 20.

71 Mediante la ord. $n^{0} 30$ del 3 de junio de 1950. Véase también Los Andes, 7/6/1950, p. 4.

72 Véase Los Andes, 17/5/1951, p. 1; 8/6/1951, p. 1 y 19/6/1951, p. 3.

73 Cf. LÓPEZ DÁVALOS; BADINO, op. cit., p. 132 y notas 28 y 29.

74 Véase Los Andes, 2/6/1954, p. 3; 4/6/1954, p. 2; 10/6/1954, p. 4; 25/6/1954, p. 2; 6/7/1954, p. 2 y 7/7/1954, p. 4.

75 Mediante res. $n^{0} 15$, del 8 de enero de 1952.

76 Cf. VERGARA BAI, Armando. Antecedentes de la evolución histórica de los recursos de uranio de la región de Cuyo entre 1951-1968 (Informe). Buenos Aires: Comisión Nacional de Energía Atómica, 1992. p. 16.

77 La información sobre las divisiones de la sección de Biología del oic, fue aportada por las entrevistas del autor al ingeniero Fidel Antonio Roig (5 de agosto de 2008), y a los doctores Armando Pisanó (4 de agosto de 2008) y Virgilio Roig (11 de noviembre de 2009).

78 Véase res. nº 198, del 29 de marzo de 1957 (AOGD-UNCuyo).

79 Véanse ord. nº 414/56, 1113/56, 847/57 y 1694/57 (AOGD-UNCuyo). También Los Andes, 5/8/1954, p. 7, 6/8/1954; p. 2, 18/12/1954; p. 4, 24/4/1956; p. 3, 11/8/1956, p. 3. y 15/9/1956, p. 4. Sobre este caso y las actividades de Enrique Brücher en el Departamento de Investigaciones Científicas véase PACHECO, Pablo Antonio. La Ahnenerbe y las actividades de Enrique Brücher en el Departamento de Investigaciones Científicas (1954-1957). Actas del IV Congreso Interoceánico de Estudios Latinoamericanos. Mendoza, 2010.

80 Véase Los Andes, 17/6/1950, p. 4.

81 La filoxera es una clase de insecto parásito que afecta a las cepas.

82 Respecto de la primera iniciativa se designa una comisión integrada por Walter Georgii, García Wimer, Arturo Corte, Santiago Curelli y Roberto Carretero, investigadores vinculados a la ingeniería, la astronomía, la meteorología y las actividades de los observatorios de San Juan y Mendoza cf. Los Andes, 5/9/1950, p. 4. Para la segunda iniciativa cf. Los Andes, 12/4/1951, p. 3.

83 Véase Los Andes, 28/4/1950, p. 3; 9/8/1950, p. 4 y 2/7/1950, p. 6.; 10/10/1950, p. 4; 11, 14 y 15/11/1950, p. 4, 3; 3, 30/11/1950, p. 3 y 5/12/1950, p. 3.

84 Véase UNIVERSIDAD NACIONAL DE CUYO. Santos Mendoza. Mendoza, p. 7.

85 Cf. ord. n 147, art. 14. En UNIVERSIDAD NACIONAL DE CUYO. La investigación en la Universidad. Mendoza, 1950. p. 27 y ord. n² 29, art. 44 (AOGDUNCuyo).

86 Véanse Anales del Departamento de Investigaciones Científicas (D.I.C.). Mendoza, tomo I, cuadernos I-II, Sección Geofísica; tomo II, fascículos 1-3, Sección Biología; tomo II, fascículos 1-4.

87 Cf. UNIVERSIDAD NACIONAL DE CUYO. Revista Matemática Cuyana. Buenos Aires, Departamento de Investigaciones Científicas, Instituto de Matemática, v. 1, fasc. 1-3; v. 2, fasc. 1-2.

88 Véase Revista Matemática Cuyana, v. 2, fasc. 2, 1956.

89 Entrevista del autor al doctor Jorge Bosch (6/6/2008). Otro miembro del instituto recuerda: “Como era previsible, Monteiro persuadió a Cruz. Pero a Cruz se le planteaba de entrada un feo problema: había un conjunto de gente que aceptaba ir a Mendoza, pero no estaba dispuesta a afiliarse al partido justicialista. Cruz se había entusiasmado mucho con el proyecto, lo suficiente como para que intentara solucionar el difícil asunto. Así que consiguió una entrevista con el propio Perón, viajó a Buenos Aires y le expuso directamente a él la cuestión. Debió haber sido extraordinariamente convincente, porque Perón, para quien alguna importancia tendría el proyecto, aceptó que, como excepción, los recalcitrantes no se afiliaran y no se diluyera la iniciativa por esa razón". KLIMOVSKY, Gregorio. Mis diversas existencias. Apuntes para una autobiografía. Buenos Aires: A-Z, 2008. p. 129.

90 Entrevista del autor al ingeniero agrónomo Fidel Antonio Roig (5/8/2008).

91 Véase Los Andes, 24/2/1955, p. 4; 13/8/1955, p. 2 y 28/8/1955, p. 2.

92 UNIVERSIDAD NACIONAL DE CUYO. Comunicaciones. Mendoza, año ॥, $2^{\mathrm{a}}$ quincena de marzo y $1^{\mathrm{a}}$ y $2^{\mathrm{a}}$ de abril, $n^{0} 45-46-47$, p. 27.

93 UNIVERSIDAD NACIONAL DE CUYO. La investigación en la Universidad. Mendoza, 1950. p. 17.

94 Mediante el decreto $\mathrm{n}^{0} 13.443$ del 4 de julio de 1950.

95 El 17 de mayo de 1951 mediante el decreto no 9.695. Véase HURTADO DE MENDOZA; BUSALA, op. cit., p. 23-26 y MYERS, op. cit., p. 108, nota 18. El autor agradece los comentarios del profesor Sergio Scalise.

96 Véase Los Andes, 7/9/1951, p. 3.

97 Comunicación personal con Enrique Oliva (noviembre de 2009).

98 Res. $n^{0} 83$, del 25 de enero de 1956 (AOGD-UNCuyo). 
Véase Los Andes, 1/11/55, p. 4 y 2/11/55, p. 4.

100 Véase RUIZ LEAL, Adrián; ROIG, Fidel A. Debe mantenerse la ciencia pura en un instituto de la Universidad Nacional de Cuyo. Ciencia e Investigación, Buenos Aires, t. 12, p. 181-182, abr. 1956.

101 Cf. entrevista del autor a los doctores Eduardo Zarantonello (21/2/208) y Virgilio Roig (11/11/2009). También KLIMOVSKY, op. cit., p. 133-134.

102 Cf. Los Andes, 3/11/1957, p. 8.

103 La prensa local se hizo eco del éxodo de profesores en ese contexto. Véase Los Andes, 15/8/1956, p. 2.

104 ROIG, Arturo Andrés. La Universidad hacia la democracia. Bases doctrinarias e históricas para la constitución de una pedagogía participativa. Mendoza: Editorial de la Universidad Nacional de Cuyo, 1998. p. 258.

105 LACOSTE, op. cit, 1997, p. 146. Véase también LACOSTE, Pablo. Utopía y resistencia (1955-1973). In: ROIG, Arturo Andrés; LACOSTE, Pablo; SATLARI,

106 Cf. MYERS, op. cit., p. 100.

[Recebido em outubro de 2010, aprovado para publicação em setembro de 2011] 